\title{
Impact of Fading on Random Access Compressed Sensing
}

\author{
Fatemeh Fazel \\ Northeastern University \\ Boston, MA \\ Email: fazel@ece.neu.edu
}

\author{
Maryam Fazel \\ University of Washington \\ Seattle, WA \\ Email: mfazel@u.washington.edu
}

\author{
Milica Stojanovic \\ Northeastern University \\ Boston, MA \\ Email: millitsa@ece.neu.edu
}

\begin{abstract}
We address the design of a large-scale sensor network, deployed for long-term environmental monitoring. Taking into account the sparse nature of the monitored field, we integrate the sensing and the communication aspects of the network into an efficient Random Access Compressed Sensing (RACS) scheme. RACS is inspired by the theory of compressed sensing and employs random channel access to collect a sufficient number of observations at the fusion center to reconstruct the field. In this paper, we study the impact of fading on RACS. We provide a framework for system design that specifically targets a Rayleigh fading channel. Moreover, we quantify the energy and bandwidth requirements and provide analytical results demonstrating the robustness of RACS in the presence of fading.
\end{abstract}

\section{INTRODUCTION}

The wireless sensor network technology has enabled affordable large coverage and long term monitoring of the environment. In a general scenario, sensor nodes are scattered over a region and monitor a slowly varying field. Each node employs a sensing scheme which dictates the frequency of measurements, then communicates its observations to a central processing unit, referred to as the fusion center (FC). Once the network is deployed, there can be little access to the sensors, hence re-charging batteries becomes difficult. This is especially of concern in underwater networks, where sensor nodes are hundreds of meters below the surface, or in wireless networks where a hostile environment prohibits access to the sensor nodes. Enabling long term deployment in such scenarios calls for energyaware sensing and communication architectures.

In [1], [2] we proposed an integrated sensing and communication architecture referred to as Random Access Compressed Sensing (RACS) which achieves overall efficiency in terms of the energy per bit of information successfully delivered. Considering the fact that most natural phenomena have a sparse representation in an appropriate domain, RACS capitalizes on integrating compressed sensing with random channel access. The former supports transmission of sensor data from only a random subset of all the nodes- thus reducing the overall energy consumption- while the latter supports a robust and simple implementation that eliminates the need for synchronization and scheduling. We studied RACS over an ideal communication channel in [1]. In the present paper, we take a step forward towards providing a system optimization methodology that is suited for realistic deployment environments where communication occurs over a fading channel. In this paper, we consider the impact of small scale fading, specifically focusing on quasi-static Rayleigh fading channels.

The rest of the paper is organized as follows. In Section II, we overview the RACS scheme. In Section III, we model the arrival of useful packet process in a fading channel. Section IV outlines a design methodology for determining the network parameters. In Section V we study the impact of fading on RACS and quantify the bandwidth and energy requirements. Finally, we provide the concluding remarks in Section VI.

\section{INTEGRATED SENSING AND COMMUNICATION}

The theory of compressed sensing [3], [4] establishes that if a signal of dimension $N$ has an $S$-sparse representation in an appropriate domain $\Psi$ (referred to as the sparsity basis), it can be recovered, with very high probability, from $\mathcal{O}(\mu S \log N)$ random measurements obtained in a sensing domain $\mathbf{\Phi}{ }^{1}$

Many natural signals have a compressible (sparse) representation in the frequency domain. Noting that the spatial coordinate basis and the spatial frequency basis are maximally incoherent, ${ }^{2}$ we employ distributed random sensing; i.e., each node senses the field at random time instants, independently of the other nodes. The sensing rate $\lambda_{1}$, which dictates the frequency of sensing per node, is thus a design parameter that fully defines the system.

We assume a lattice network structure where $N$ sensors are placed on a two-dimensional grid. The sensor located at position $(i, j)$ measures the physical quantity of interest $u_{i j}$ at a rate of $\lambda_{1}$ measurements per second. The node encodes each measurement along with its location tag into a packet of $L$ bits. This data packet is then modulated and transmitted to the FC in a random access fashion. Because of the nature of random access, packets from different nodes may collide at the FC. The FC simply discards the packets that are distorted and collects the remaining useful packets over an observation interval $T$. This interval is assumed to be shorter than the coherence time of the process, such that the process can be approximated as fixed during $T$. It suffices to ensure that the FC collects a minimum number of packets $N_{s}=C S \log N$ picked uniformly at random from different sensors, to guarantee accurate reconstruction of the field with very high probability. Note that $C$ is a constant that is independent of $S$ and $N$ (see [1], [2] for details of the RACS model).

\section{ARrival of Useful Packets}

As a simple model, we assume that each node generates packets according to an independent Poisson process at an average rate of $\lambda_{1}$ packets per second. In the absence of fading, two factors contribute to packet loss, collisions and repetitions. The former refers to the overlapping of packets at the FC, while the latter refers to a situation in which the same sensor transmits more than one packet during a given collection interval. In case of repetitions, if the FC has successfully received a packet from a given node, it discards repetitions since they do not provide any new information. Note that if a given packet is lost due to collision, a repetition may still be received successfully. When fading is present, not every collision

${ }^{1}$ The coefficient $\mu$ represents the coherence between the sparsity basis $\Psi$ and the sensing basis $\boldsymbol{\Phi}$ and is defined as [5]

$$
\mu(\boldsymbol{\Phi}, \boldsymbol{\Psi})=\sqrt{N} \max _{1 \leq k, j \leq N}\left|\left\langle\boldsymbol{\Phi}_{k}, \boldsymbol{\Psi}_{j}\right\rangle\right|
$$

${ }^{2}$ The coherence between the (spatial) Fourier domain $\boldsymbol{\Psi}$ and the spatial domain $\boldsymbol{\Phi}$ is $\mu(\boldsymbol{\Psi}, \boldsymbol{\Phi})=1$ (see [5]). 
results in packet loss. Namely, it is possible that although two (or more) packets overlap at the FC, one is sufficiently stronger so that it can be successfully detected, i.e., it can capture the receiver.

The total number of packets that are used in the reconstruction process, $K\left(\lambda_{1}, T\right)$, is the number of received packets left after discarding the erroneous and repetitive packets. A packet is declared erroneous if it does not pass the cyclic redundancy check (CRC) or a similar verification procedure. The FC buffers the useful packets and attempts reconstruction at the end of the observation interval $T$. It then moves on to the next interval. In a given interval, reconstruction will be successful if $N_{s}$ or more useful packets are collected. Otherwise, reconstruction for that particular interval will fail.

In order to determine the probability distribution of the number of useful packets $K\left(\lambda_{1}, T\right)$, we start with modeling the impact of fading, and move on to model the impact of repetitions.

\section{A. Modeling the Impact of Rayleigh Fading}

A wireless communication channel is modeled by distancedependent propagation loss, shadowing and small-scale fading [6]. The total channel gain $c_{i}$, observed by the $i$-th packet, can thus be modeled as

$$
c_{i}=G_{i} h_{i}
$$

where small scale fading is modeled by the complex Gaussian coefficient $h_{i} \sim \mathcal{C N}(0,1)$ and $G_{i}$ includes the effects of path loss and shadowing. We assume that the path loss and shadowing are pre-compensated at the transmitter, by means of a power control mechanism, which can be achieved through an occasional downlink beacon. Thus the transmitter adjusts its power to $P_{0} / G_{i}$, where $P_{0}$ is the design value of the average received power. The received power of packet $i$ is then

$$
P_{R_{i}}=\left|c_{i}\right|^{2} P_{0} / G_{i}^{2}=\left|h_{i}\right|^{2} P_{0}
$$

Denoting the packet of interest by index 0 , the instantaneous signalto-interference ratio (SIR) is given by

$$
\gamma=\frac{P_{R_{0}}}{\sum_{i=1}^{n} P_{R_{i}}}
$$

where $n$, the number of interfering packets, is a random variable with probability distribution $P(n)$. For the sake of simplicity, we have assumed here that the powers of all $n$ interfering packets are added, even though they each may be only partially overlapping with the desired packet. Note that for the system parameters of interest, $P(n)$ is negligible for $n>1$, thus the above assumption leads only to an insignificant overestimation of the effect of interference, yielding a safety margin. Successful transmission of a packet, referred to as capture, is an event in which either (a) there is no interference to the desired packet (i.e., $n=0$ ), or (b) interference is present but the power of the desired packet is sufficiently greater than the total interfering power, i.e., $\gamma>b$, where, $b>1$ is a pre-defined threshold with a typical value $b=2-6$ [7], [8].

The probability of capture is thus given by

$$
p_{c}=P(0)+\sum_{n=1}^{N-1} P(n) \operatorname{Prob}\left\{\frac{P_{R_{0}}}{\sum_{i=1}^{n} P_{R_{i}}}>b\right\}
$$

where $P(n)$ is the probability that there are $n$ interfering packets. Assuming that the packet generation follows a Poisson distribution, we have that

$$
P(n)=\frac{\left(2 N \lambda_{1} T_{p}\right)^{n}}{n !} e^{-2 N \lambda_{1} T_{p}}
$$

where $T_{p}=L / B$ is the packet duration, with $L$ representing the number of bits per packet and $B$ the transmission bandwidth. ${ }^{3}$ From [7, p. 369] we have that

$$
\operatorname{Prob}\left\{\frac{P_{R_{0}}}{\sum_{i=1}^{n} P_{R_{i}}}>b\right\}=(b+1)^{-n}
$$

Hence, from Eqs. (5) and (6), the probability of capture is obtained as

$$
p_{c}=P(0)+e^{-2 N \lambda_{1} T_{p}} \sum_{n=1}^{N-1} \frac{\left(2 N \lambda_{1} T_{p}\right)^{n}}{n !} \cdot \frac{1}{(b+1)^{n}}
$$

When $N$ is large and $2 N \lambda_{1} T_{p} \sim 1$ which is the case for the system parameters of interest to us, Eq. (7) is well approximated by

$$
p_{c}=e^{-2 N \lambda_{1} T_{p} \beta}
$$

where

$$
\beta=\frac{b}{b+1}
$$

In a non-fading channel, $\beta=1$ (equivalently, $b \rightarrow \infty$ ) and Eq. (8) corresponds to the probability of no collision [1].

\section{B. Packet Repetitions}

To assess the impact of duplicate packets, we focus on an individual node. Packets generated by a particular node either capture the receiver (with probability $p_{c}$ given by Eq. (8)) or fail to capture it (with probability $1-p_{c}$ ). For a particular node, let $M_{1}(T)$ denote the number of packets generated during $T$ that have captured the receiver. If this number is greater than or equal to 1 , the FC will keep one successfully delivered packet and discard any other packets received from that node. Now the probability of receiving a useful packet, $p_{g}$, can be expressed as

$$
p_{g}=\operatorname{Prob}\left\{M_{1}(T) \geq 1\right\}=\sum_{l=1}^{m} \frac{\left(\lambda_{1} T\right)^{l}}{l !} e^{-\lambda_{1} T}\left[1-\left(1-p_{c}\right)^{l}\right]
$$

where the first term is the probability that the node generates $l$ packets during $T$, and the second term is the probability that one or more of the generated packets capture the channel. Note that since a node does not interfere with its own packet, the maximum number of packets that are generated by a single node during $T$ is given by $m=\left\lfloor\frac{T}{T_{p}}\right\rfloor \gg 1$. The expression (10) thus reduces to

$$
p_{g}=1-e^{-p_{c} \lambda_{1} T}
$$

The average number of useful packets received from a given node during $T$ is equal to $p_{g}$. Thus the average effective arrival rate of packets at the $\mathrm{FC}$ is

$$
\lambda^{\prime}=\frac{N p_{g}}{T}=\frac{N}{T}\left(1-e^{-p_{c} \lambda_{1} T}\right)
$$

where $p_{c}$ is given by Eq. (8).

\section{Arrival of Useful Packets}

The total number of packets that are used in the reconstruction, $K\left(\lambda_{1}, T\right)$, equals the number of packets that have captured the receiver, excluding duplicates. We assume that the arrival of useful packets follows a Poisson process ${ }^{4}$ with an effective average arrival rate $\lambda^{\prime}$ given by Eq. (12). The Poisson model for $K\left(\lambda_{1}, T\right)$ thus

\footnotetext{
${ }^{3}$ Without loss of generality, throughout this paper we use bandwidth and bit rate interchangeably, i.e., we assume a BPSK modulation.

${ }^{4}$ For analyzing random access protocols, it is commonly assumed that the aggregate packet arrival, including retransmitted packets in case of collisions, follows a Poisson model [7].
} 
follows as

$$
\operatorname{Prob}\left\{K\left(\lambda_{1}, T\right)=k\right\}=P_{K}\left(k ; \lambda_{1}, T\right)=\frac{\left(\lambda^{\prime} T\right)^{k}}{k !} e^{-\lambda^{\prime} T}
$$

\section{SYSTEM DESIGN}

With the analytical model (13) at hand, we proceed to determine the sensing rate necessary to achieve a desired performance metric. Towards this end, we define a performance requirement based on the concept of sufficient sensing.

\section{A. Probability of Sufficient Sensing}

We define the probability of sufficient sensing as the probability that the FC collects $N_{s}$ or more useful packets during $T$, and we specify the performance requirement as the minimum probability of sufficient sensing, $P_{s}$. Thus

$$
\operatorname{Prob}\left\{K\left(\lambda_{1}, T\right) \geq k\right\} \geq P_{s}
$$

where $P_{s}$ is the desired probability of sufficient sensing (which is a system design parameter). Let $\alpha=\lambda^{\prime} T$ represents the average number of useful packets collected in $T$. For a given $N_{s}$ and a desired probability of sufficient sensing $P_{s}$, one can find the corresponding $\alpha_{s}$ to meet the sufficient sensing requirement. In other words, the condition (14) can equivalently be stated as

$$
\alpha \geq \alpha_{s}
$$

Fig. 1 shows the $\alpha_{s}$ required to achieve a desired probability of sufficient sensing, versus the size of the network $N$. Assuming the sparsity $S$ is fixed, we note that the growth rate of $\alpha_{s}$ versus $N$ is fairly low: While $N$ grows 100 -fold from $N=10^{3}$ to $N=10^{5}$, $\alpha_{s}$ grows only by a factor of 1.6. This observation will be used in Section V-C to illustrate that the energy consumption increases fairly slowly with the size of the network.

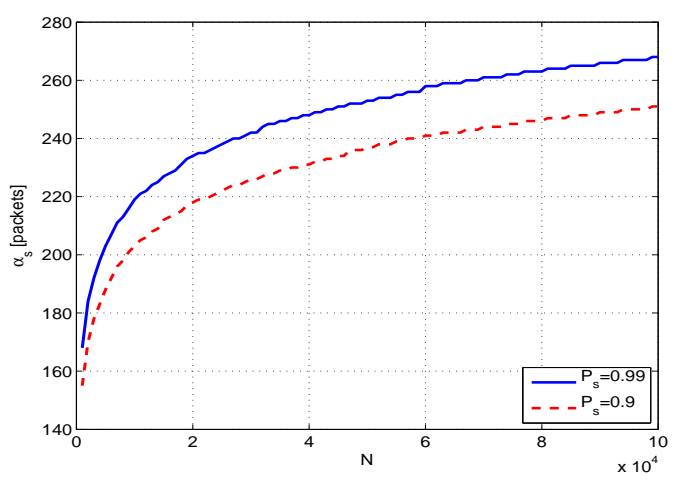

Fig. 1. The average number of useful packets required, $\alpha_{s}$, vs. the size of the network $N(S=10)$.

\section{B. Design Objective}

The design objective is to determine the per-node sensing rate $\lambda_{1}$ that is necessary to ensure sufficient sensing. Condition (15) implies that

$$
\lambda_{1 s} \leq \lambda_{1} \leq \lambda_{1 c}
$$

where $\lambda_{1 s}$ and $\lambda_{1 c}$ are the solutions to $\alpha=\alpha_{s}$ illustrated in Fig. 2. We are only interested in those values of $\lambda_{1}$ for which the system is stable, i.e., for which an increase in $\lambda_{1}$ results in an increased number of useful packets. Thus the desired value of the per-node sensing rate lies in the stable region

$$
\lambda_{1 s} \leq \lambda_{1} \leq \lambda_{1 m}
$$

where $\lambda_{1 m}$ is the point at which $\alpha$ reaches its maximum value $\alpha_{\max }$. By setting the derivative of $\alpha$ with respect to $\lambda_{1}$ equal to zero, $\lambda_{1 m}$ is obtained as

$$
\lambda_{1 m}=\frac{1}{T} \log \frac{1}{1-\alpha_{s} / N}
$$

The desired operating point is chosen to be at the lower edge of the stable region, i.e., at $\lambda_{1 s}$, since a lower per-node sensing rate corresponds to lower energy consumption, as we will discuss in Section V-C. The per-node sensing rate $\lambda_{1 s}$ can be expressed in terms

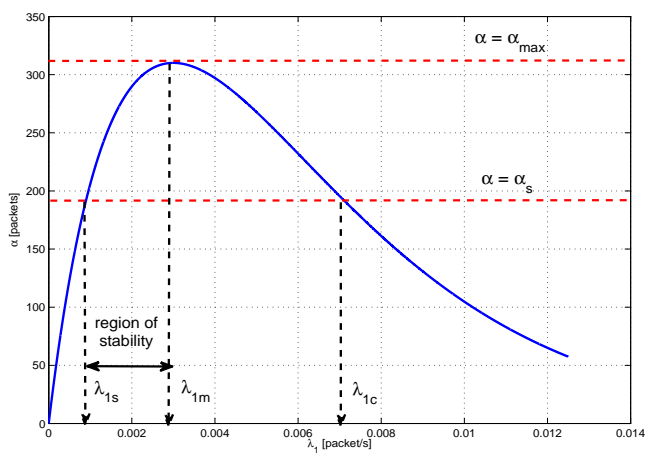

Fig. 2. The average number of useful packets $\alpha$ vs. the per-node packet generation rate $\lambda_{1}$ plotted for $N=2500, L=1000$ bits, $B=10 \mathrm{kbps}$, $T=120 \mathrm{~s}$ and $b=2$. Shown in the figure are $\lambda_{1 s}$ and $\lambda_{1 c}$ (solutions to $\left.\alpha=\alpha_{s}\right)$ and the region of stability $\left(\lambda_{1 s}, \lambda_{1 m}\right)$.

of $\alpha_{s}$ as

$$
\lambda_{1 s}=\frac{-1}{2 N T_{p} \beta} \cdot W_{0}\left(\frac{2 N T_{p} \beta}{T} \log \left(1-\frac{\alpha_{s}}{N}\right)\right)
$$

where $W_{0}(\cdot)$ denotes the principal branch of the Lambert $\mathrm{W}$ function. ${ }^{5}$ For example, for $\alpha_{s}=174$ and $\alpha_{s}=188$, the corresponding minimum per-node sensing rates are $\lambda_{1 s}=6.3 \times 10^{-4}$ packet/s and $\lambda_{1 s}=6.9 \times 10^{-4} \mathrm{packet} / \mathrm{s}$, respectively.

\section{Performance Evaluation}

In this section we assess the impact of fading and show that RACS is robust to fading. We then quantify the bandwidth and energy requirements.

\section{A. Robustness Against Fading}

In the absence of fading, the effective packet arrival rate is determined as

$$
\lambda_{\text {non-fading }}^{\prime}=\frac{N}{T}\left(1-e^{-\lambda_{1} T e^{-2 N \lambda_{1} T_{p}}}\right)
$$

Note that the model (20) is a slight improvement upon the model in [1] since in (20) we have exploited the repetitive packets to enhance the performance, whereas in [1] we discarded repetitions. Comparing the model (20) with that of Eq. (12), we note that

$$
\lambda_{\text {non-fading }}^{\prime} \leq \lambda^{\prime}
$$

${ }^{5}$ The Lambert $\mathrm{W}$ function $W(x)$ is defined by

$$
W(x) e^{W(x)}=x \quad \text { for } \quad x \geq-1 / e
$$

The branch satisfying $W(x) \geq-1$ is referred to as the principal branch of the Lambert $\mathrm{W}$ function and is denoted by $W_{0}(x)$. 
Fig. 3 shows $\alpha$ plotted versus $\lambda_{1}$. Both fading and non-fading scenarios, as given by Eqs. (12) and (20), are shown. Employing the same per-node sensing rate, the average number of useful packets received over a fading channel is greater than that in a non-fading channel. This suggests that RACS is robust against fading, i.e., if a system is designed to perform in non-fading channel conditions, in the event such a system undergoes fading, not only does it not suffer performance loss, the performance actually improves. In other words, the system now achieves sufficient sensing with a probability greater than the targeted $P_{s}$.

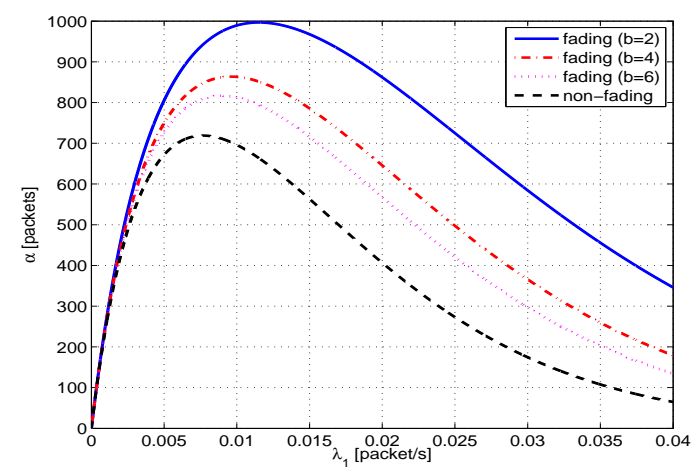

Fig. 3. The average number of useful packets $\alpha$ received in $T=120 \mathrm{~s}$ vs. $\lambda_{1}$, for $N=2500, L=1000$ bits and $B=38.4$ kbps. Note that $\lambda_{1 s}^{\text {fading }} \leq \lambda_{1 s}^{\text {non fading }}$.

\section{B. Bandwidth Utilization}

In bandwidth-limited networks, the bandwidth $B$ required by each sensor to communicate its data arises as a figure of merit for system performance. The minimum bandwidth $B_{s}$ required to achieve a certain performance over fading channels, corresponds to the case in which there is only a single solution to $\alpha=\alpha_{s}$, i.e., when $\lambda_{1 s}=\lambda_{1 c}=\lambda_{1 m}$ with $\lambda_{1 m}$ given by Eq. (18). Let us define $x_{s}$ as

$$
x_{s}=\frac{2 N T_{p} \beta}{T} \log \left(1-\frac{\alpha_{s}}{N}\right)
$$

In order to have a valid solution for $\lambda_{1 s}$, Eq. (19) implies that $W_{0}\left(x_{s}\right)$ has to be negative. Thus

$$
-1 \leq W_{0}\left(x_{s}\right) \leq 0
$$

In (23), the limit $W_{0}\left(x_{s}\right)=0$ is achieved when $x_{s}=0$, or equivalently, when $B \rightarrow \infty$. The other limit, $W(x)=-1$, is achieved when $x_{s}=-1 / e$ which corresponds to the minimum bandwidth. The minimum bandwidth $B_{s}$ is thus obtained as

$$
B_{s}=\frac{2 e N L \beta}{T} \log \left(\frac{1}{1-\alpha_{s} / N}\right)
$$

Thus $B_{s}$ in a fading channel is lower than its non-fading counterpart by a factor of $\beta$, i.e.,

$$
\frac{B_{s}}{B_{s, \text { non-fading }}}=\beta<1
$$

Fig. 4 shows the minimum bandwidth versus $N$ for a fading as well as a non-fading channel.

\section{Energy Consumption}

In a battery-powered network where lifetime is of utmost importance, energy per successfully delivered bit of information constitutes

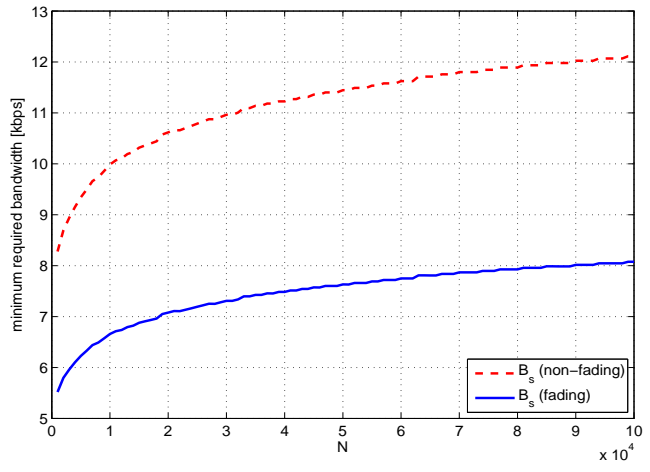

Fig. 4. Minimum required bandwidth $B_{s}$ for fading and non-fading channels, for $S=10, L=1000$ bits, $T=120 \mathrm{~s}, b=2$ and $P_{s}=0.99$.

a figure of merit for system performance. Let us denote by $P_{T}$ the average power that a node consumes for transmission, i.e.,

$$
P_{T}=P_{0} E\left\{G_{i}^{-2}\right\}=P_{0} K
$$

where the expectation taken with respect to shadow fading as well as the distance. Determining $K$ in general depends on the geometry of the region and the placement of the FC. The total average energy required for one field reconstruction is

$$
E=N \lambda_{1} T \cdot P_{T} \cdot T_{p}
$$

where the first term $\left(N \lambda_{1} T\right)$ is the average number of nodes that transmit in one collection interval $T$. As expected, for a given bandwidth $B$, the energy consumption is minimized if one chooses the minimum sensing rate $\lambda_{1 s}(B)$, i.e.,

$$
E_{\min }(B)=N \lambda_{1 s}(B) T \cdot P_{T} \cdot L / B
$$

We note that the energy expenditure $E_{\min }$ depends on the transmission bandwidth $B$ through the two parameters, $\lambda_{1 s}$ and $T_{p}$, both of which are decreasing functions of $B$. Hence,

$$
E_{\min }(\infty) \leq E_{\min }(B) \leq E_{\min }\left(B_{s}\right)
$$

We note that $\lambda_{1 s}(B)$ is largest when $B=B_{s}$ and decreases with $B$, reaching a limiting value $\lambda_{1 s}(\infty)$ as $B \rightarrow \infty$. Thus for a given $B$,

$$
\lambda_{1 s}(\infty) \leq \lambda_{1 s}(B) \leq \lambda_{1 s}\left(B_{s}\right) \quad \text { for } B \geq B_{s}
$$

The lower and upper limits of $\lambda_{1 s}(B)$ are analytically derived as

$$
\lambda_{1 s}(\infty)=\frac{1}{T} \log \frac{1}{1-\alpha_{s} / N}
$$

and

$$
\lambda_{1 s}\left(B_{s}\right)=\frac{e}{T} \log \frac{1}{1-\alpha_{s} / N}
$$

Correspondingly, the lower and upper bounds on the energy expenditure are given as

$$
E_{\text {low }}=\lim _{B \rightarrow \infty} E_{\min }(B)=0
$$

and

$$
E_{\mathrm{up}}=E_{\min }\left(B_{s}\right)=\frac{1}{2 \beta} P_{T} T
$$

Note that $E_{\text {low }}=0$ is an artifact of fixed transmission power $P_{T}$. In a practical system, however, the per-node transmission power will be set so as to achieve a certain SNR; consequently the transmission power will grow with the bandwidth $B$. For large $N$, the energy can 
be approximated as

$$
E_{\min }(B) \approx \frac{-1}{2 T_{p} \beta} \cdot W_{0}\left(\frac{-2 T_{p} \beta \alpha_{s}}{T}\right) \cdot T \cdot T_{p} \cdot P_{T}
$$

Noting that $W_{0}(x) \leq x$ we have that

$$
E_{\min }(B) \leq \alpha_{s} \cdot T_{p} \cdot P_{T}
$$

Hence as $N \rightarrow \infty$ the energy consumption grows linearly with $\alpha_{s}$. In Fig. 1 we observed that the rate of increase of $\alpha_{s}$ is small compared to that of $N$. Thus, as shown in Fig. 5, for a given $B$ the energy expenditure barely grows with the size of the network $N$. For example, increasing the size of the network from $N=10^{3}$ to $N=10^{5}$, results in only $2 \mathrm{~dB}$ increase in the overall energy consumption. This fact demonstrates the power of RACS. Fig. 6

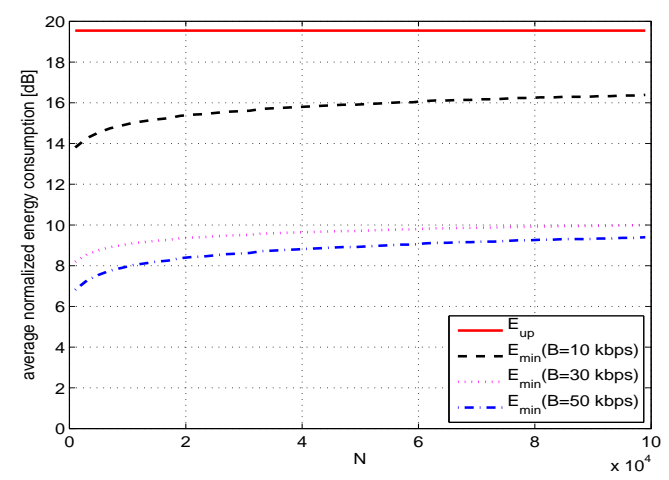

Fig. 5. Average energy consumption vs. the number of nodes $N$. The upper bound on the energy consumption (given by Eq. (34)) is also shown.

compares the energy expenditure of the RACS network normalized by $P_{T}$ in fading and non-fading channels. We observe that the energy consumption in a fading channel is lower than that of a non-fading channel, due to the lower per-node sensing rate.

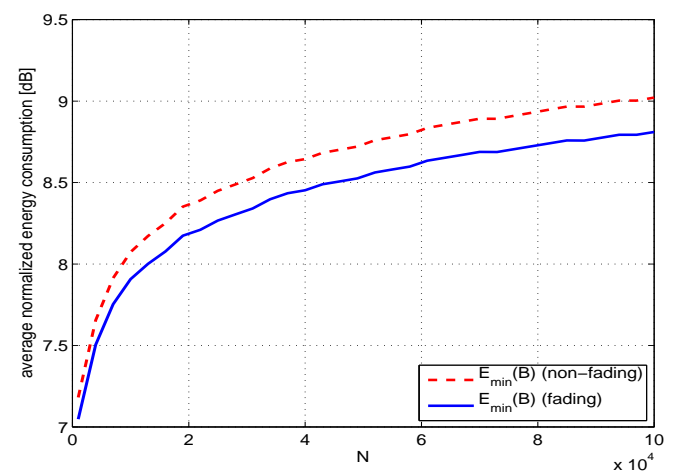

Fig. 6. Average energy consumption vs. the number of nodes $N$.

\section{Energy and Bandwidth Savings of RACS}

Finally, to demonstrate the usefulness of the RACS scheme, we compare the energy and bandwidth requirements of RACS with those of a conventional (benchmark) design in fading. The benchmark scheme is a TDMA network in which all $N$ nodes transmit using pre-assigned time slots. The bandwidth and energy requirements of a TDMA network are given by [1]

$$
B_{s, T D M A}=\frac{N L}{T}
$$

and

$$
E_{T D M A}=N T_{p} P_{T}
$$

The savings in bandwidth and energy achieved by RACS are, respectively,

$$
G_{B}=\frac{1}{2 e \beta \log \frac{1}{1-\alpha_{s} / N}}
$$

and

$$
G_{E}=\frac{-2 N T_{p} \beta}{W_{0}\left(\frac{2 N T_{p} \beta}{T} \log \left(1-\frac{\alpha_{s}}{N}\right)\right) T}
$$

These savings are shown in Fig. 7.
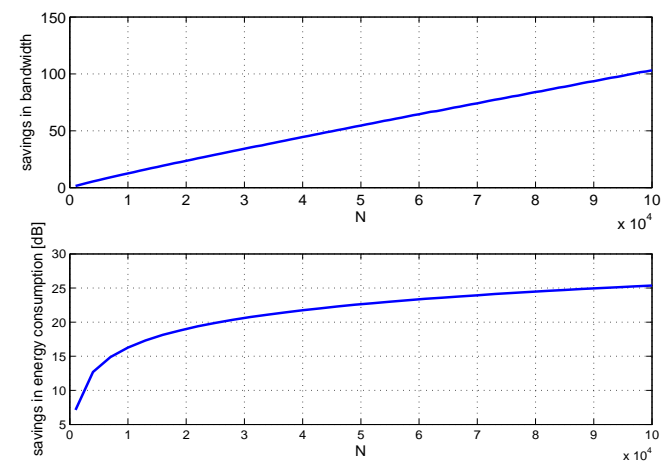

Fig. 7. Savings in bandwidth and energy requirements with respect to a benchmark TDMA network. The system parameters are $P_{s}=0.99, S=10$, $L=1000$ bits, $T=120 \mathrm{~s}$ and $b=2$.

\section{CONCLUSION}

RACS is an integrated sensing and communication architecture that combines the concepts of random channel access and compressed sensing to achieve energy and bandwidth efficiency. In this paper, we took into consideration the impact of channel fading on the design and performance of RACS. We provided system design optimization for a Rayleigh fading channel and assessed the performance of RACS analytically in terms of the energy and bandwidth utilization. Compared to a system operating over a non-fading channel, we showed that fading boosts the performance of RACS, or equivalently it requires lower bandwidth and energy to achieve the same probability of sufficient sensing.

\section{REFERENCES}

[1] F. Fazel, M. Fazel, and M. Stojanovic, "Compressed sensing in random access networks with applications to underwater monitoring," Physical Communication (Elsevier) Journal, 2011.

[2] _ _ "Random access compressed sensing for energy-efficient underwater sensor networks," IEEE Journal on Selected Areas in Communications (JSAC), vol. 29, no. 8, Sept. 2011.

[3] E. J. Candes, J. Romberg, and T. Tao, "Robust uncertainty principles: Exact signal reconstruction from highly incomplete frequency information," IEEE Transactions on Information Theory, vol. 52, pp. 489-509, March 2006.

[4] E. Candes and J. Romberg, "Sparsity and incoherence in compressive sampling," Inverse Problems, vol. 23, pp. 969-985, 2006.

[5] E. J. Candes and M. B. Wakin, "An introduction to compressive sampling," IEEE Signal Processing Magazine, pp. 21-30, March 2008.

[6] A. Goldsmith, Wireless Communications. Cambridge University Press, 2005.

[7] M. Schwartz, Mobile Wireless Communications. Cambridge University Press, 2005.

[8] J. Linnartz, Narrowband Land-Mobile Radio Networks. Artech House, Inc., 1993. 